\title{
Evaluation of the quantitative prediction of a trend reversal on the Japanese stock market in 1999.
}

\author{
Anders Johansen ${ }^{1}$ and Didier Sornette Sh, $^{1,2,3}$ \\ ${ }^{1}$ Institute of Geophysics and Planetary Physics \\ University of California, Los Angeles, California 90095 \\ ${ }^{2}$ Department of Earth and Space Science \\ University of California, Los Angeles, California 90095 \\ ${ }^{3}$ Laboratoire de Physique de la Matière Condensée \\ CNRS UMR6622 and Université de Nice-Sophia Antipolis \\ B.P. 71, Parc Valrose, 06108 Nice Cedex 2, France
}

October 26, 2018

\begin{abstract}
In January 1999, the authors published a quantitative prediction that the Nikkei index should recover from its 14 year low in January 1999 and reach $\approx 20500$ a year later. The purpose of the present paper is to evaluate the performance of this specific prediction as well as the underlying model: the forecast, performed at a time when the Nikkei was at its lowest (as we can now judge in hindsight), has correctly captured the change of trend as well as the quantitative evolution of the Nikkei index since its inception. As the change of trend from sluggish to recovery was estimated quite unlikely by many observers at that time, a Bayesian analysis shows that a skeptical (resp. neutral) Bayesian sees her prior belief in our model amplified into a posterior belief 19 times larger (resp. reach the $95 \%$ level).
\end{abstract}

keywords: Stock market; Log-periodic oscillations; scale invariance; prediction; Gold; Nikkei; Herding behaviour. 
Following the general guidelines proposed in [1], the authors made in January 1999 public through the Los Alamos preprint server [2] a quantitative prediction stating that the Nikkei index should recover from its 14 year low (actually 13232.74 on 5 Jan 1999 ) and reach $\approx 20500$ a year later corresponding to an increase in the index of $\approx 50 \%$. Furthermore, this prediction was mentioned in a wide-circulation journal which appeared in May 1999 [3].

Specifically, based on a third-order "Landau" expansion

$$
\frac{d F(\tau)}{d \log \tau}=\alpha F(\tau)+\beta|F(\tau)|^{2} F(\tau)+\gamma|F(\tau)|{ }^{4} F(\tau) \ldots
$$

in terms of $\tau \equiv t-t c$, where $t_{c}=31$ Dec. 1989 is the time of the all-time high of the Nikkei index, the authors arrived at the equation

$$
\begin{gathered}
\log (p(t)) \approx A^{\prime}+\frac{\tau^{\alpha}}{\sqrt{1+\left(\frac{\tau}{\Delta_{t}}\right)^{2 \alpha}+\left(\frac{\tau}{\Delta_{t}^{\prime}}\right)^{4 \alpha}}} \\
\left\{B^{\prime}+C^{\prime} \cos \left[\omega \log \tau+\frac{\Delta_{\omega}}{2 \alpha} \log \left(1+\left(\frac{\tau}{\Delta_{t}}\right)^{2 \alpha}\right)+\frac{\Delta_{\omega}^{\prime}}{4 \alpha} \log \left(1+\left(\frac{\tau}{\Delta_{t}^{\prime}}\right)^{4 \alpha}\right)+\phi\right]\right\},
\end{gathered}
$$

describing the time-evolution of the Nikkei Index $p(t)$. Equation (22) was then fitted to the Nikkei index in the time interval from the beginning of 1990 to the end of 1998, i.e., a total of 9 years. Extending the curve beyond 1998 thus provided us with a quantitative prediction for the future evolution of the Index. In figure 1, we compare the actual and predicted evolution of the Nikkei over 1999. We see that not only did the Nikkei experience a trend reversal as predicted, but it has also followed the quantitative prediction with rather impressive precision, see figure 2. It is important to note that the error between the curve and the data has not grown after the last point used in the fit. This tells us that the prediction has performed well so far. Furthermore, since the relative error between the fit and the data is within $\pm 2 \%$ over a time period of 10 years, not only has the prediction performed well, but also the underlying model. This analysis represents the correct quantitative evaluation of the performance of the model as well as its predictive power on the Nikkei Index over a quite impressive time-span of 10 years.

We wish to stress that the fulfilling of our prediction is even more remarkable than the comparison between the curve and the data indicates. This, since it included a change of trend: at the time when the prediction was issued, the market was declining and showed no tendency to increase. Many economists were at that time very pessimistic and could not envision when Japan and its market would rebounce. For instance, the well-known economist P. Krugman [4] wrote July 14, 1998 in the Shizuoka Shimbun at the time of the banking scandal "the central problem with Japan right now is that there just is not enough demand to go around - that consumers and corporations are saving too much and borrowing too little... So seizing these banks and putting them under more responsible management is, if anything, going to further reduce spending; it certainly will not in and of itself stimulate the economy... But at best this will get the economy back to where it was a year or two ago - that is, depressed, but not actually plunging." Then, in the Financial Times, January, 20th, 1999, P. Krugman wrote in 
an article entitled "Japan heads for the edge" the following: "...the story is starting to look like a tragedy. A great economy, which does not deserve or need to be in a slump at all, is heading for the edge of the cliff - and its drivers refuse to turn the wheel." In a poll of thirty economists performed by Reuters (the major news and finance data provider in the world) in October 1998 [5], only two economists predicted growth for the fiscal year of 1998-99. For the year 1999-2000 the prediction was a meager $0.1 \%$ growth. This majority of economists said that "a vicious cycle in the economy was unlikely to disappear any time soon as they expected little help from the government's economic stimulus measures... Economists blamed moribund domestic demand, falling prices, weak capital spending and problems in the bad-loan laden banking sector for dragging down the economy."

Nevertheless, we predicted $\mathrm{a} \approx 50 \%$ increase of the market in the next 12 months assuming that the Nikkei would stay within the error-bars of the fit. At the time of writing (3rd February 2000 ), the market is up by $\approx 49.5 \%$ and the error between the prediction and the curve has not increased, see figure 2. Predictions of trend reversals is noteworthy difficult and unreliable, especially in the linear framework of auto-regressive models used in standard economic analyses. The present nonlinear framework is well-adapted to the forecasting of change of trends, which constitutes by far the most difficult challenge posed to forecasters. Here, we refer to our prediction of a trend reversal within the strict confine of equation (2): trends are limited periods of times when the oscillatory behavior shown in figure 11 is monotonous. A change of trend thus corresponds to crossing a local maximum or minimum of the oscillations.

We report one case. In the standard "frequentist" approach to probability [6] and to the establishment of statistical confidence, this bears essentially no weight and should be discarded as story telling. We are convinced that the "frequentist" approach is unsuitable to assess the quality of such a unique experiment as presented here of the prediction of a global financial indicator and that the correct framework is Bayesian. Within the Bayesian framework, the probability that the hypothesis is correct given the data can be estimated, whereas this is excluded by construction in the standard "frequentist" formulation, in which one can only calculate the probability that the null-hypothesis is wrong, not that the alternative hypothesis is correct (see also [7] for recent introductory discussions).

Bayes' theorem states that

$$
P\left(H_{i} \mid D\right)=\frac{P\left(D \mid H_{i}\right) \times P\left(H_{i}\right)}{\sum_{j} P\left(D \mid H_{j}\right) P\left(D_{j}\right)} .
$$

where the sum in the denominator runs over all the different conflicting hypothesis. In words, equation (3) estimates that the probability, that hypothesis $H_{i}$ is correct given the data $D$, is proportional to the probability $P\left(D \mid H_{i}\right)$ of the data given the hypothesis $H_{i}$ multiplied with the prior belief $P\left(H_{i}\right)$ in the hypothesis $H_{i}$ divided with the probability of the data. In the present context, we use only the two hypotheses $H_{1}$ and $H_{2}$ that our prediction of a trend reversal is correct or that it is wrong. For the data, we take the change of trend from bearish to bullish. We now want to estimate whether the fulfillment of our prediction was a "lucky one". We quantify the general atmosphere of disbelief that Japan would recover by the value $P\left(D \mid H_{2}\right)=5 \%$ for the probability that the Nikkei will change trend while disbelieving our model. We assign the classical confidence level of $P\left(D \mid H_{1}\right)=95 \%$ for the probability that the 
Nikkei will change trend while believing our model.

Let us consider a skeptical Bayesian with prior probability (or belief) $P\left(H_{1}\right)=10^{-n}, n \geq 1$ that our model is correct. From (3), we get

$$
P\left(H_{1} \mid D\right)=\frac{0.95 \times 10^{-n}}{0.95 \cdot 10^{-n}+0.05 \times\left(1-10^{-n}\right)} .
$$

For $n=1$, we see that her posterior belief in our model has been amplified compared to her prior belief by a factor $\approx 7$ corresponding to $P\left(H_{1} \mid D\right) \approx 70 \%$. For $n=2$, the amplification factor is $\approx 16$ and hence $P\left(H_{1} \mid D\right) \approx 16 \%$. For large $n$ (very skeptical Bayesian), we see that her posterior belief in our model has been amplified compared to her prior belief by a factor 0.95/0.05 = 19. Alternatively, consider a neutral Bayesian with prior belief $P\left(H_{1}\right)=1 / 2$, i.e., a priori she considers equally likely that our model is correct or wrong. In this case, her prior belief is changed into the posterior belief equal to

$$
P\left(H_{1} \mid D\right)=\frac{0.95 \cdot \frac{1}{2}}{0.95 \cdot \frac{1}{2}+0.05 \cdot \frac{1}{2}}=95 \% .
$$

This means that this single case is enough to convince the neutral Bayesian.

We stress that this specific application of Bayes' theorem only deals with a small part of the model, i.e., the trend reversal. It does not establish the significance of the quantitative description of 10 years of data (of which the last one was unknown at the time of the prediction) by the proposed model within a relative error of $\approx \pm 2 \%$.

A question that remains is how far into the future will the Japanese stock market continue to follow equation (2)? Obviously, the Nikkei Index must "break away" at some point in the future even if there are no changes in the overall behaviour and the underlying model thus remains valid. The reason is that the prediction was made using a third order expansion. This means that, as the parameter $\tau=t-t_{c}$ in equation (2) continues to increase, this approximation becomes worse and worse and a fourth order term should be included. Presently, we are not ready to present the derivation of such an equation. Furthermore, we expect the numerical difficulties involved in fitting an even more complex equation than equation (2) to be considerable.

Last, we would like to bring to the attention of the reader that not only can bearish markets occasionally be described by the framework underlying equation (2). In fact, bullish markets exhibits such changes of regimes even more frequently, see [8].

Acknowledgement The authors wish to thank D. Stauffer for his encouragement both with respect to the original work of [2] as well as the present re-evaluation.

\section{References}

[1] A. Johansen and D. Sornette, Modeling the Stock Market prior to large crashes, Eur. Phys. J. B, 9, 167-174. Available on http://www.nbi.dk/ johansen/pub.htm 
[2] The prediction was made public on the 25 Jan. 1999 by posting a preprint on the Los Alamos server, see http://xxx.lanl.gov/abs/cond-mat/9901268. The preprint was later published as A. Johansen and D. Sornette, Financial "anti-bubbles": log-periodicity in Gold and Nikkei collapses, Int. J. Mod. Phys. C. 10, 563-575 (1999).

[3] D. Stauffer, Monte-Carlo-Simulation mikroskopischer Börsenmodelle, Physikalische Blätter 55 (1999) 49.

[4] The Official Paul Krugman Web Page: http://web.mit.edu/krugman/www/

[5] Reported in Indian Express on the 15 Oct., see http://www.indian-express.com/fe/daily/19981016/28955054.html

[6] H. Jeffreys, Theory of Probability, 3rd ed. (Oxford University Press, 1961)).

[7] D. Malakoff, Bayes Offers a 'New' Way to Make Sense of Numbers, Science 286, 1460-1464 (1999); A Brief Guide to Bayes Theorem, ibid 286, 1461 (1999); G. D'Agostini, G., Teaching statistics in the physics curriculum: Unifying and clarifying role of subjective probability, Am. J. Phys. 67, 1260-1268 (1999).

[8] A. Johansen, D. Sornette and O. Ledoit, Predicting Financial Crashes Using Discrete Scale Invariance, J. of Risk, Vol 1 No. 4, pp.5-32 (1999). Available on http://www.nbi.dk/ johansen/pub.htm; A. Johansen and D. Sornette, Log-periodic power law bubbles in LatinAmerican and Asian markets and correlated anti-bubbles in Western stock markets: An empirical study. Subm. to J. Empirical Finance. Preprint available on http://www.nbi.dk/ johansen/pub.htm 


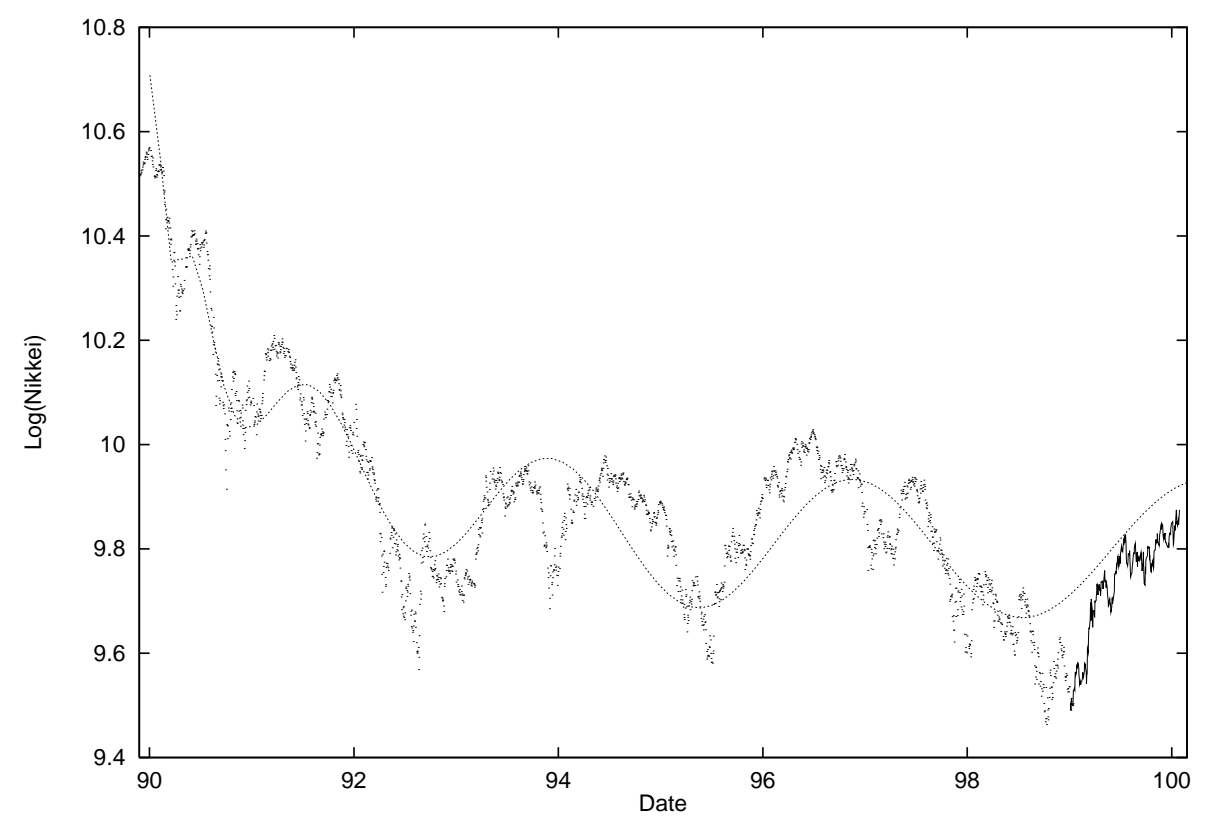

Figure 1: . Logarithm of the Nikkei Index compared to equation 2. The dots are the data used in the fit of equation (2) being the ticked line and covers the 9 year period from 31 Dec. 1989 to 31 Dec. 1998. The solid line is the actual behaviour of the Nikkei after the last point used in the fit and covers the period 1 Jan. 1999 to 28 Jan. 2000. The prediction was made public on the 25 Jan. 1999 [2]. See [2] for details of the fit.

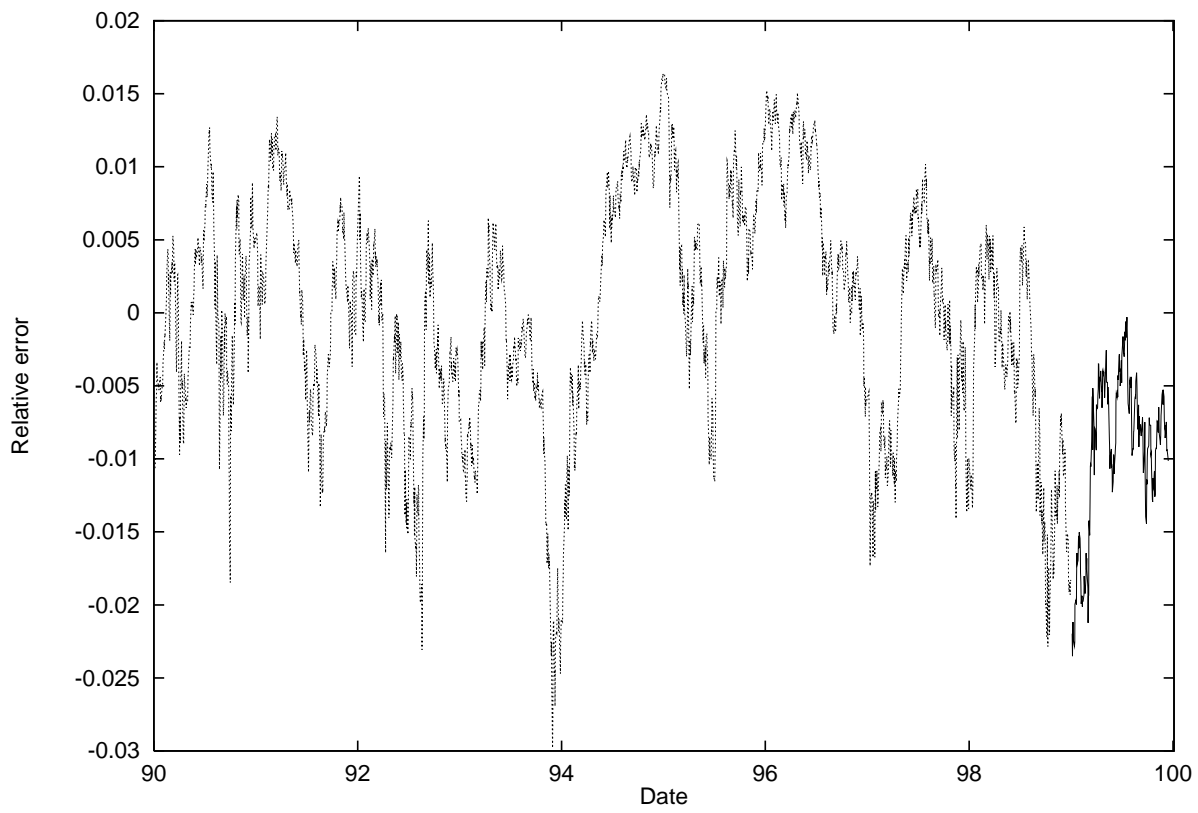

Figure 2: The relative error between the fit with equation (2) and the data. The ticked line if the relative error between the fit and the data used in the fit. The solid line is the error between the prediction and the actual data. 